\title{
Coronary computed tomographic angiography studies: A brief review in analysis, interpretation, and reporting
}

\author{
Teuku Yusrizal ${ }^{1,3}$, Ivana Purnama Dewi ${ }^{1,2,3}$, Rosi Amrilla Fagi ${ }^{1,3}$ \\ ${ }^{1}$ Faculty of Medicine, Universitas Airlangga, Surabaya, Indonesia \\ ${ }^{2}$ Faculty of Medicine, Duta Wacana Christian University, Yogyakarta, Indonesia \\ ${ }^{3}$ Department of Cardiology and Vascular Medicine, Dr. Soetomo General Hospital, Surabaya, Indonesia
}

Received: 2021-01-09.

Accepted: 2021-02-06

This work is licensed under a

Creative Commons Attribution 4.0 International License

J Clin Med Kaz 2021; 18(2):4-7

Corresponding author:

Teuku Yusrizal.

E-mail: t.yusrizalxx06@gmail.com;

ORCID: 0000-0001-7336-2799

\begin{abstract}
Coronary computed tomographic angiography is a non-invasive procedure that makes it very convenient to imagine the anatomical ability to remove coronary artery disease in patients with chest pain in a population at low to moderate risk. For plaque identification and physiological assessment, coronary computed tomographic angiography may be used, facilitating thorough diagnostic and prognostic assessment of primary prevention in patients. Overall plaque load is assessed by the coronary computed tomographic angiography, plaque subtypes are distinguished and high-risk plaque is identified. The purpose of this article is to review existing applications and practices related to the use of automatic techniques in coronary computed tomographic angiography analysis and interpretation.
\end{abstract}

Key words: coronary computed tomographic angiography, CTA, coronary artery disease, CAD, imaging studies

\section{Introduction}

With the introduction of radiological methods, the diagnostic approach to cardiac and cardiovascular disease is increasingly evolving. Coronary Computed Tomographic Angiography (CTA) is a non-invasive procedure that enables atherosclerotic stenosis of the epicardial coronary arteries to be specifically visualized anatomically, with minimal radiation exposure. Although the cumulative assessment can be restricted by conditions such as elevated heart rate, obesity, arrhythmia, and high coronary calcium load, major technical advancements in cardiac imaging allow for action to be acquired with higher spatial precision in some instances. The diagnostic precision of coronary CTA has been found to be superior to invasive coronary angiography (ICA), and has recently become the preferred reference for coronary artery disease (CAD) assessment [1,2].

Despite advances in revascularization and pharmacotherapy, CAD complications are also one of the world's leading causes of death. Myocardial infarction with acute segment elevation and sudden coronary death requires new approaches in detection and prevention. Vulnerable plaque (VP) is a term based on the idea that ultimately a prospective detectable precursor lesion will evolve into an acute coronary syndrome (ACS) plaque.

To recognize VP, a variety of imaging modalities and clinical biomarkers have been studied over time. The use of ICA only focuses on measuring the degree of coronary stenosis (luminography), while coronary CTA will observe the lumen and walls of the coronary arteries noninvasively, thus gaining details on the existence, severity, and features of CAD, including atherosclerotic plaque and visualization of luminal obstruction. This is an interesting addition to the medical diagnostic tools currently available, such as imaging for nuclear perfusion or invasive selective coronary angiography. In addition, coronary CTA could help us to avoid PCI in the event of CAD disorders in small vessels and to initiate optimal and aggressive medical therapy in cases of extensive nonobstructive CAD [1-3].

Our aim is here to discuss the Coronary CTA studies focused in analysis, interpretation, and reporting. The articles in this review have been selected from mainly Embase and Pubmed, published in the last 10 years, through keywords, such as computed tomography angiography, CTA, coronary artery disease, interpretation and reporting. 


\section{Scanning technique}

Automatic coronary CTA is conducted in 3 stages by the COR Analyzer algorithm; coronary artery segmentation, coronary artery labelling, and identification of stenosis. The lungs are observed as an air-filled region that is segmented using a morphological filter in the initial stage. A circular hough transform is used to detect the ascending aorta which is visible at the axial slice in the circular portion of the mediastinum. In comparison to the surrounding structure, it is often segmented using $3 \mathrm{D}$ active surface minimization, based on the distinct edges of the filled aorta.

The coronary artery is segmented by following a contrastfilled region connected to the ascending aorta and traced through the tubular component attached to it. The coronary arteries are then labeled using a probabilistic model that trains the algorithm using the CTA study guide. The major arteries, such as the the right coronary artery (RCA) left main artery (LM), the left anterior descending artery (LAD), and the left circumflex artery (LCx), are labeled and checked on the basis of the arterial spatial relationship (Figure 1). The area where the main artery is absent would be read as an incomplete segmentation if there is a difference in the arterial anatomy, as in the arterial anomaly [4].

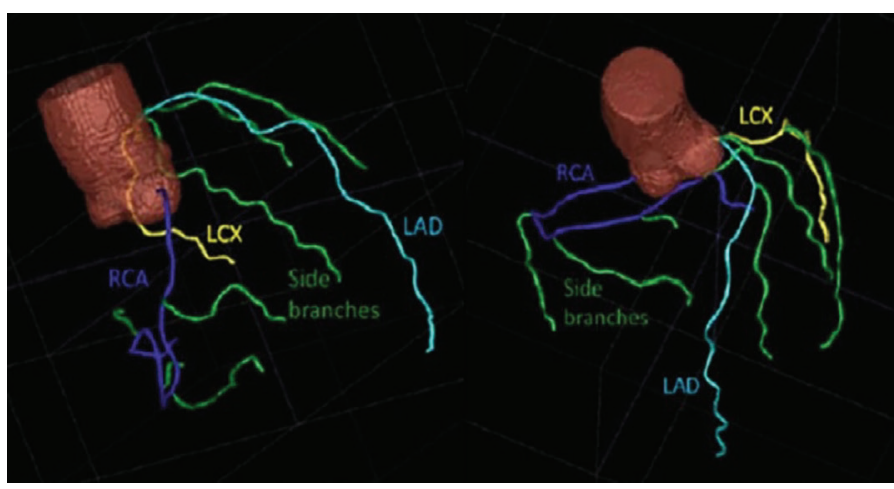

Figure 1. The Cor A algorithm, which includes automated segmentation, midline extraction, and branch marking, displays the right anterior and left anterior oblique of the coronary artery branches and main aorta [4]

Since the blood vessels are separated into discontinuous parts, the labeled coronary arteries are then analysed. Using a literary model-based variation, the lumens and walls are represented. Hyestersis-based adaptive binarization has been used to detect and segment calcified lesions. Between the lumen and vessel wall, non-calcified plaques were found as hypodense areas without calcium. For cross-sectional area (of blood vessels and lumen, plaque, artifact, branching, and distance from the ostium, each segment was analyzed. The characteristic lesions used to train the algorithm are then matched against these characteristics. Throughout the reformation of the multiplanar curves created automatically along the vessel diameter, detection marks are placed over large stenotic areas $(>50 \%)$ and warning signs are placed over segments of possible processing failure [4].

A validation test to validate the accuracy of the procedure is conducted at each stage in the algorithm. If the confidence level of the findings is poor, the device will announce a mistake or alert if an issue prevails in the study portion. It also evaluates the adequacy of vascular tracking, sudden absence of the blood vessel, imaging artifacts, the risk of dropping the vessel toward a single coronary artery, and the traced vessel's inadequate coverage of the AV groove. It is calculated that the average time for all the steps listed above is 5-7 minutes [4].

\section{Principles of coronary CTA interpretation}

The original source image needs to be reconstructed. The resulting transverse image can then be used to perform the following post-processing techniques:

\section{a. Multi-planar reformations (MPR).}

Show a data set from a three-dimensional space in every imaging plane. The MPR is easy to reconstruct and can be stored for further study and archived into the PACS (image database and communication system). If rebuilt along the diameter of the vessel (ie "curved" MPR), the long, tortuous coronary artery can be represented in a single image. Curved MPR is considered very important for the diagnosis, imaging, and illustration of CAD because it is usually tortuous and complicated to view on a 2D image. The curved MPR allows a single image to be shown of the whole blood vessel or section of the coronary artery, allowing a full illustration of coronary artery stenosis (5). In all general post-processing processes, MPR can be reconstructed quickly and is mandatory for the diagnosis of CAD because coronary stenosis must be validated in order to prevent false positive outcomes [5].

\section{b. Maximum intensity projections (MIP).}

Displays the highest volume voxel attenuation against the display that offers an angiographic image. Thus, without the need for complicated modifications of many parameters, MIP allows quick reconstruction. Maximum intensity projections can, however, lead to overestimation of coronary stenosis due to calcified plaque and should not be used without thin-section MPR cross-examination to confirm the diagnosis [5].

\section{c. Volume rendering technique (VRT).}

Utilizes the entire data set so as to provide a threedimensional view of anatomy. VRT can be used to show coronary anatomy if there is coronary artery anomaly. In addition, the patient's veins after bypass surgery can be well illustrated using the VRT technique. VRT, similar to MIP, should not be used to assess coronary stenosis, because VRT images are dependent on several parameters and coronary stenosis can easily be exaggerated or underestimated [5].

\section{d. Virtual Intravascular Endoscopy (VIE).}

In order to provide a $3 \mathrm{D}$ view of anatomy, the whole data set is used. Where there is a coronary artery anomaly, VRT can be used to explain coronary anatomy. Moreover, using the VRT method, the patient's veins following bypass surgery can be well demonstrated. In order to test coronary stenosis, VRT, similar to MIP, should not be used because VRT images rely on many criteria and coronary stenosis can easily be exaggerated or underestimated [6].

\section{e. Cine Imaging.}

Cine imaging is used to display cardiac features such as the left ventricle and heart valves for motion and physiological characteristics. $40 \%$ may indicate end-systole depending on the particular patient, and $90 \%$ may equate to end-diastole, with the remaining data points representing other stages of the cardiac cycle. Cine photographs are known to be very helpful for analyzing left ventricular wall displacement and thickening of the wall and for measuring valve motion in several phases [6].

For the measurement of coronary CTA images, normal sagittal, coronal, and transverse, imaging fields are typically not appropriate since the coronary arteries mostly run oblique and transverse to these planes (Figure 2). The following standard areas are found useful for the assessment of coronary anatomy:

a. The short-axis plane provides a perpendicular view of $L A D$ and a longitudinal view of $L C x$ and $R C A$

$b$. The orthogonal long-axis planes provide perpendicular views of the proximal and distal portions of $L C x$ and RCA, also 


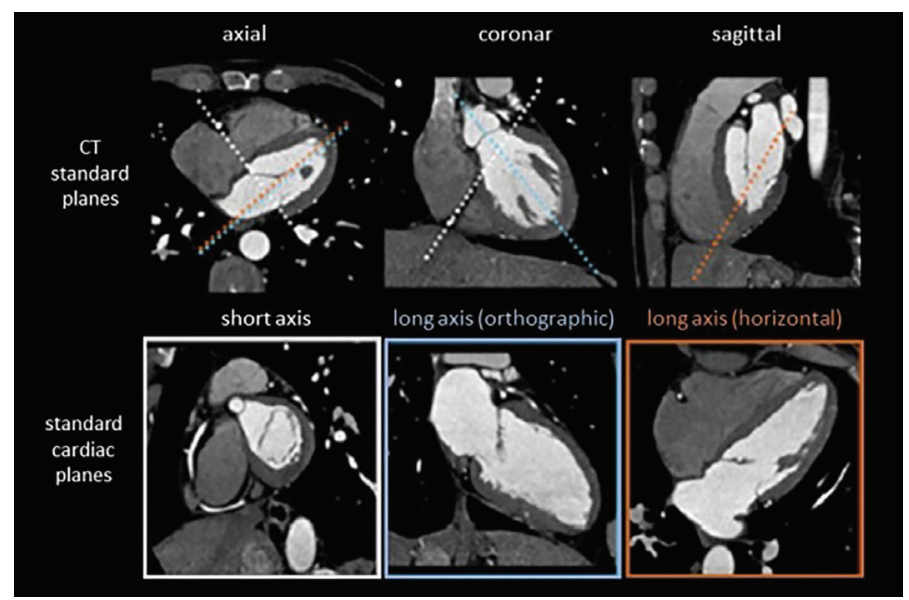

Figure 2. Guidelines for the adjustment of the field of imaging from the standard CT plane to the standard cardiac plane [7].

longitudinal views of $L A D$, the posterior descending artery $(P D A)$, and the central portion of $L C x$ and $R C A$

c. The horizontal long-axis planes provide a perpendicular view of the center of $L C X$ and $R C A$, and a longitudinal view of distal RCA, PDA, and LAD.

\section{Analysis and interpretation}

Non-contrast examinations of the heart are routine but a risk assessment should be performed in asymptomatic individuals. In non-contrast tests, Coronary Calcium Score computer programs usually classify pixels above $130 \mathrm{HU}$ as the calcium corresponding amount. In any vessel distribution (right coronary artery, left descending anterior and circumflex artery), the reader should classify each lesion. A scoring software based on volumetric calculation or the area density (Agatston score) of each calcified focus would produce a cumulative score for each vessel $[7,8]$.

Mass scores are used less frequently in clinical practice. As there is no validity evidence for these interventions at present. The use of mass scores should often be followed by the more standard (and scientifically recognized) reporting of the Agatston score. Other existing calcium measurement approaches contribute to the same questions. Of all coronary beds, the gross coronary calcium score is the number of all calcified lesions. The calcium in the annulus or mitral valve, aortic valve, aorta, and pericardium or myocardium are removed from the overall coronary calcium ratings [7].

Calcium score report depends on the preferences of the reader, with the total calcium score being reported and the percentiles compared with the nomogram for age and sex. Additionally, calcium should be registered (but not counted) in other areas of the heart. As the recommended but optional reporting tool, the aortic valve, mitral ring, and aortic wall may be semi-quantified (mild, moderate, or extreme calcifications) since these measurements can have separate prognostic and diagnostic significance [7].

Guiding principles for interpretation include: detailed multi-plane and cross-sectional examination of each coronary portion, detection of related objects, evaluation of lesion morphology and structure, also evaluation of stenosis severity using high-resolution images (including MPR format) in both transverse and longitudinal blood vessel views. Viewing images of the sagittal and coronal planes can assist in the identification of artifacts [7].

Figure 3 is a standard model that, by defining minor modifications, was modified for coronary CTA (7). Compared

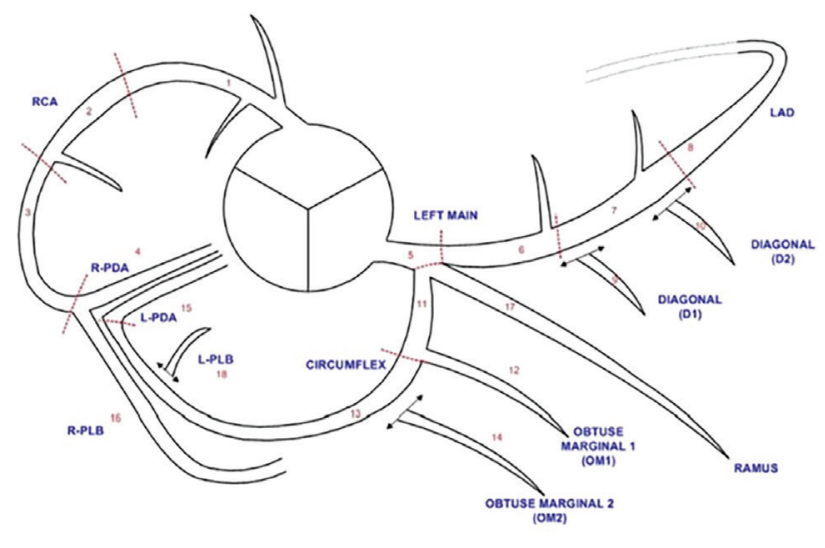

Figure 3. Coronary segmentation diagram by Society of Cardiovascular Computed Tomography (SCCT) [7].

to the standard view acquired during the ICA, this model was modified to more accurately resemble the appearance of the CTA. This model differs from the standard 1975 AHA segmentation in the following respects, in addition to integrating 3 standard invasive angiographic views into a single axial view: the ramus intermedius branch was added as segment 17 and the left posterolateral branch is defined as segment 18 [7].

For routes and branching of the main coronary arteries and their divisions, the coronary tree should initially be studied. With regard to their origin, pathway, and connection to essential structures such as the chambers of the heart, interventricular septum, aorta, and pulmonary arteries, coronary abnormalities should be examined. The overall elasticity and caliber of the coronary artery lumen should be checked. Variations of CT density can be observed in the mural and intraluminal sections of the coronary artery and contrasted with the neighboring interstitial lumen and calcified density containing contrast, such as bone or calcified plaque [7].

In order to assess the vulnerability of the myocardium as a whole, atherosclerotic lesions must be considered in relation to their segmental location. The effect of the luminal plaque should be measured in terms of the maximum percentage yield of stenotic diameter. Other steps are additive but not necessary, such as minimum luminal area and percent stenosis area. Because the presence of remodelled intramural plaques can be positively visualized by coronary CTA and noncalcified, partly noncalcified and calcified plaques can be distinguished, this feature can also be analyzed and segmentally reported $[7,8]$.

As low CT density (HU) levels do not necessarily correlate closely with anatomical or biochemical pathology, the classification of plaque as "noncalcified" is preferred over "soft" or "lipid-rich." When image quality is appropriate, it is recommended that plaque morphological features such as ulceration, dissection, and fissure be considered. 'Ostial', 'branch', 'long' and 'positive remodeling' are additional optional plaque updates. Such entities, such as coronary aneurysms, should carry out non-cardiac thoracic evaluation studies into other related artery pathologies [7].

\section{Report the findings of cardiac CTA imaging}

There is a recommended standard template for disclosing coronary CTA results. It is important to consider the inclusion of a schematic image as a description of the most appropriate imaging results [5]:

a. The calcium assessment result should state the distribution (diffuse or localized) and degree of calcification 
(massive or nodular) in each coronary artery (eg, RCA, LAD and $L C x$ ) should be stated.

b. Subsequently, the presence or absence of anomalous coronary arteries should be identified in coronary anatomy, as well as the form of coronary artery supply (left dominant, right dominant, or balanced form).

c. Each coronary artery is viewed separately with respect to the presence and type of plaque (ie, absence of calcification, mixture, or presence of calcification).

d. The key information for the coronary CTA results section is the semi-quantitative documentation of the degree of stenosis using the words mild (less than 30\% luminal narrowing), moderate (30-50\%), moderate to extreme (50-75\%), and extreme stenosis (>75\%). The origin, path and origin, position of the anastomosis, and position and grade of the stenotic graft, should be mentioned if a coronary artery bypass graft is present. It is important to take caution to make notes on the risk of jump anastomosis.

e. Next, other non-coronary heart findings such as valve morphology, aortic roots, size of pulmonary and arterial trunks, atrial and ventricular septum, and the presence of myocardial disease.

f. Finally, it is important to indicate extracardiac findings in the mediastinum, lung, and chest wall, including image examination of the upper abdomen.

\section{Conclusion}

Coronary artery disease (CAD) is a major cause of mortality and morbidity in developed countries. Coronary CTA provides both the patient and the referral with many benefits by non-invasively offering a precise and detailed measurement of the coronary arteries and vessel walls. A consistent and comprehensive approach to evaluating and understanding coronary CTA studies is needed for the steady rise in examinations being carried out worldwide.

Recent technologies in CT technology enhance coronary CTA image quality, viability, and precision. To build and maintain an effective coronary CTA method, a thorough knowledge of the technique's strengths and limitations, and an awareness of the specifics of patient planning, scanning procedures, and image restoration are required.

Disclosures: There is no conflict of interest for all authors. Acknowledgements: None.

Funding: None.

Author contribution: TY and RAF conceived the idea and designed the report. TY were a major contributor in writing the manuscript. TY and IPD prepare the final manuscript for publication.

\section{References}

1. Carità P, Guaricci AI, Muscogiuri G, Carrabba N, Pontone G. Prognostic value and therapeutic perspectives of coronary CT angiography: A literature review. Biomed Res Int. 2018;. doi:10.1155/2018/6528238

2. Goldstein JA. Coronary CT Angiography: Identification of Patients and Plaques “At Risk”. J Am Coll Cardiol. 2018; 71(22):25232526. doi:10.1016/j.jacc.2018.02.080

3. Kuchynka P, Lambert L, Černý V, Marek J, Ambrož D, Danek BA, et al. Coronary CT angiography. Cor et Vasa. 2015; 57(6):e425-e432. doi:10.1016/j.crvasa.2015.09.008

4. Rajiah P, Schoenhagen P. Automated Interpretation and Reporting of Coronary CT Coronary Angiography. Curr Cardiovasc Imaging Rep. 2013; 6(3):282-291. doi:10.1007/s12410-013-9201-9

5. Karlo CA, Leschka S, Stolzmann P, Glaser-Gallion N, Wildermuth S, Alkadhi H. A systematic approach for analysis, interpretation, and reporting of coronary CTA studies. Insights Imaging. 2012; 3(3):215-228. doi:10.1007/s13244-012-0167-y

6. Sun Z. Coronary Computed Tomographic Angiography in Coronary Artery Disease: A Systematic Review of Image Quality, Diagnostic Accuracy, and Radiation Dose. New York: Nova Science Publishers, Inc; 2013.

7. Leipsic J, Abbara S, Achenbach S, Cury R, Earls JP, et al. SCCT guidelines for the interpretation and reporting of coronary CT angiography: A report of the Society of Cardiovascular Computed Tomography Guidelines Committee. J Cardiovasc Comput Tomogr. 2014; 8(5):342-358. doi:10.1016/j.jcct.2014.07.003

8. Abbara S, Blanke P, Maroules CD, Cheezum M, Choi AD, Han BK, et al. SCCT guidelines for the performance and acquisition of coronary computed tomographic angiography: A report of the Society of Cardiovascular Computed Tomography Guidelines Committee Endorsed by the North American Society for Cardiovascular Imaging (NASCI). J Cardiovasc Comput Tomogr. 2016; 10:435-449. doi: 10.1016/j.jcct.2016.10.002 